\title{
Impacto das estratégias adotadas para enfrentar a pandemia de COVID-19 em um Instituto Brasileiro de referência em cirurgia de alta complexidade em Ortopedia e Traumatologia*
}

\section{Impact of the Strategies Adopted to Face the COVID-19 Pandemic in a Brazilian Reference Institute for High Complexity Surgery in Orthopedics and Traumatology}

\author{
Geraldo da Rocha Motta Filho ${ }^{10}$ Ana Carolina Leal ${ }^{2}$ Marcus Vinicius Galvão do Amaral ${ }^{3}$ \\ Phelippe Augusto Valente Maia ${ }^{4}$ Maria Eugênia Leite Duarte ${ }^{2}$ Germana Lyra Bähr ${ }^{5}$
}

\footnotetext{
${ }^{1}$ Divisão de Ensino e Pesquisa, Instituto Nacional de Traumatologia e Ortopedia, Rio de Janeiro, RJ, Brasil

2 Divisão de Ensino e Pesquisa, Instituto Nacional de Traumatologia e Ortopedia, Rio de Janeiro, RJ, Brasil

${ }^{3}$ Divisão de Ortopedia e Traumatologia, Instituto Nacional de

Traumatologia e Ortopedia, Rio de Janeiro, RJ, Brasil

${ }^{4}$ Unidade Hospitalar, Instituto Nacional de Traumatologia e

Ortopedia, Rio de Janeiro, RJ, Brasil

5 Programas Especiais, Instituto Nacional de Traumatologia e

Ortopedia, Rio de Janeiro, RJ, Brasil
}

Rev Bras Ortop 2021;56(2):161-167.

\section{Resumo \\ Palavras-chave \\ - COVID-19 \\ - coronavírus \\ - SARS-CoV-2 \\ - trauma \\ - ortopedia \\ - cirurgia}

Endereço para correspondência Geraldo da Rocha Motta Filho, MD, MSc, Rua Raimundo de Magalhães, 92, Rio de Janeiro, RJ, 22451-150, Brasil (e-mail: geraldomotta@terra.com.br).

\footnotetext{
Estudo desenvolvido na Divisão de Ensino e Pesquisa, Instituto Nacional de Traumatologia e Ortopedia, Rio de Janeiro, RJ, Brasil.
}

recebido

16 de Dezembro de 2020

aceito

11 de Fevereiro de 2021
A pandemia de COVID-19 exigiu reorganização e adaptação substanciais dos serviços de saúde em todo o mundo. Este estudo tem como objetivo analisar o efeito das estratégias operacionais implementadas no Brasil em resposta à pressão extra imposta aos serviços de saúde pela pandemia de COVID-19 de 2020. Esta pesquisa examina principalmente a estratégia de conversão de um instituto especializado em procedimentos ortopédicos eletivos de alta complexidade em uma unidade de trauma para todos os pacientes com traumatismo musculoesquelético de toda uma unidade federativa. Um estudo retrospectivo comparou as variáveis hospitalares no período de pico da pandemia (de 16 de março de 2020 a 30 de junho de 2020) com o mesmo período de 2019, que representou os valores basais. As variáveis analisadas foram número de profissionais afastados do trabalho, cirurgias realizadas, atendimento
DOI https://doi.org/ $10.1055 / \mathrm{s}-0041-1728703$. ISSN $0102-3616$. (c) 2021. Sociedade Brasileira de Ortopedia e Traumatologia. All rights reserved.

This is an open access article published by Thieme under the terms of the Creative Commons Attribution-NonDerivative-NonCommercial-License, permitting copying and reproduction so long as the original work is given appropriate credit. Contents may not be used for commercial purposes, or adapted, remixed, transformed or built upon. (https://creativecommons.org/ licenses/by-nc-nd/4.0/)

Thieme Revinter Publicações Ltda., Rua do Matoso 170, Rio de Janeiro, RJ, CEP 20270-135, Brazil 


\begin{abstract}
Keywords

- COVID-19

- coronavirus

- SARS-CoV-2

- trauma

- orthopedics

- surgery

COVID-19 pandemics required substantial reorganization and adaptation of healthcare services all over the world. This study aims to analyze the effect of operational strategies implemented in Brazil to manage the extra strain placed on healthcare services by the COVID-19 pandemic of 2020. In particular, this investigation examines the strategy to convert an institute specialized in elective orthopedic procedures of high complexity into a trauma unit for all musculoskeletal trauma patients of an entire federative unit. A retrospective study was conducted comparing hospital variables at the peak period of the pandemic (from March 16, 2020 to June 30, 2020) with the same period in 2019 as a comparative baseline. The variables analyzed included number of professionals away from work, surgeries performed, outpatient care, transfers, length of stay, number of patients diagnosed with COVID-19 and patient mortality. During the COVID-19 peak period, there was a $48.5 \%$ reduction in surgical productivity and $72.4 \%$ reduction in outpatient care compared with the same period in 2019. The number of transfers increased substantially (124.5\%), while 94 confirmed cases and 77 suspected cases of COVID-19 were reported. The mortality rate increased by $245 \%$. The present study highlighted the effect of COVID-19 on a tertiary orthopedic hospital. Despite the dramatic changes in hospital operations, due to the implementation of protocols to manage the pandemic, the results demonstrated the feasibility and efficiency of such protocols in prioritizing quality and safety for patients and the healthcare workforce.
\end{abstract}

ambulatorial, transferências, tempo de internação, número de pacientes com diagnóstico de COVID-19 e mortalidade dos pacientes. Durante o período de pico de COVID-19, houve uma redução de $48,5 \%$ na produtividade cirúrgica e de $72,4 \%$ no atendimento ambulatorial em comparação ao mesmo período de 2019. O número de transferências aumentou de maneira substancial (124,5\%), com relato de 94 casos confirmados e 77 casos suspeitos de COVID-19. A taxa de mortalidade aumentou 245\%. Este estudo destacou o efeito da COVID-19 em um hospital ortopédico terciário. Apesar das mudanças dramáticas no funcionamento do hospital devido à instituição de protocolos em resposta à pandemia, os resultados demonstraram a viabilidade e a eficiência de tais protocolos em priorizar a qualidade e a segurança dos pacientes e dos profissionais de saúde.

\section{Introdução}

Em 12 de março de 2020, a Organização Mundial da Saúde (OMS) declarou a uma pandemia global de COVID-19, a doença causada pelo vírus SARS-CoV-2, modificando diversos aspectos da vida em todo o mundo. ${ }^{1}$

No Brasil, o primeiro caso de COVID-19 foi confirmado em São Paulo, em 26 de fevereiro de 2020. Logo em seguida, o Rio de Janeiro notificou seu primeiro caso, em 5 de março de 2020; em 17 de março de 2020, o estado do Rio de Janeiro já apresentava transmissão comunitária da doença, levando à declaração de estado de emergência e à introdução de medidas de contenção da progressão da COVID-19. Apesar dessas medidas, a doença se espalhou rapidamente, atingindo seu pico em maio de 2020 e mantendo um elevado índice nacional de novos casos e óbitos. Ao final de junho, haviam sido registrados 1.408 .485 casos e 59.656 óbitos, com 10.080 casos confirmados no estado do Rio de Janeiro. ${ }^{2}$ Todos os estados brasileiros passaram por um período de alta demanda por exames diagnósticos, equipamentos de proteção individual (EPIs) e leitos hospitalares nos meses de abril e maio de 2020. Essa demanda, porém, foi atendida com dificuldade apesar da construção de hospitais de campanha para atendimento exclusivo de pacientes com COVID-19.

Embora a ortopedia e a traumatologia, como especialidades médicas, não lidem diretamente com os efeitos causados pelo SARS-CoV-2, seu desempenho foi bastante afetado devido à suspensão dos procedimentos eletivos pelas autoridades de saúde. A medida, adotada em todo o mundo, foi motivada pelo alto risco de exposição de pacientes e profissionais de saúde ao vírus e pela necessidade de alocar recursos e infraestrutura hospitalar para o tratamento das vítimas da pandemia. ${ }^{3-5}$

A maioria dos estados brasileiros reorganizou seus serviços de saúde para liberar leitos de hospitais gerais e de emergência e priorizar pacientes com COVID-19. Em cada estado, isso levou à transferência de todos os casos de trauma ortopédico para um único hospital especializado em procedimentos ortopédicos eletivos de alta complexidade. 
O objetivo deste estudo é avaliar o efeito das estratégias operacionais no contexto da pandemia, em especial na conversão de um instituto especializado em procedimentos ortopédicos eletivos de alta complexidade em uma unidade de atendimento a pacientes com traumatismos musculoesqueléticos. Os protocolos e estratégias descritos no artigo podem ser facilmente aplicados a outras unidades de saúde, sendo de grande utilidade no enfrentamento da segunda onda da doença.

\section{Métodos}

Este estudo retrospectivo avalia o efeito das medidas tomadas para o manejo da pandemia de COVID-19 nas atividades de um hospital ortopédico de alta complexidade no Brasil. Os dados foram coletados a partir de informações do sistema de gestão de dados institucionais (Portal MV e MV) e das atas das reuniões do Gabinete de Crise institucional. Este estudo foi aprovado pelo Comitê de Ética em Pesquisa da instituição.

De acordo com as orientações das autoridades sanitárias e órgãos de controle, os procedimentos eletivos foram suspensos, com realização apenas de tratamentos oncológicos, de traumas ortopédicos, infecções e complicações pós-operatórias. Além disso, o hospital passou a ser responsável pelo atendimento de todos os pacientes com traumatismo ortopédico de seu estado. Para se adaptar à nova rotina, as estratégias e protocolos elaborados foram colocados em prática e sua implementação foi coordenada pelo Gabinete de Crise por meio de reuniões virtuais. As decisões foram comunicadas aos colaboradores por meio de mensagens veiculadas por WhatsApp, televisores institucionais localizados em áreas de grande concentração de colaboradores e pela intranet, à qual todos os colaboradores tiveram acesso. Os novos protocolos implementados a partir de 16 de março de 2020 foram relacionados à reorganização das atividades institucionais, recepção e encaminhamento de pacientes transferidos e reorganização dos procedimentos cirúrgicos. Essas áreas constituem o escopo deste estudo.

\section{Reorganização das Atividades Institucionais}

As ações relacionadas à reorganização das atividades institucionais abrangeram as atividades assistenciais e acadêmicas e são resumidas na - Tabela 1.

\section{Recepção e Encaminhamento de Pacientes Transferidos}

Devido à impossibilidade de realização da reação da cadeia da polimerase (PCR) em swabs de nasofaringe e/ou orofaringe de todos os pacientes, os fluxos e protocolos de entrada dos pacientes transferidos precisaram ser definidos. $\mathrm{O}$ primeiro grupo, de pacientes assintomáticos, foi acomodado em área específica. O segundo grupo, composto por casos suspeitos de COVID-19 (ou seja, com histórico de possível contágio ou sintomas semelhantes aos da gripe) foram colocados em uma segunda área com 40 leitos; o terceiro grupo, formado por pacientes transferidos internados com diagnóstico confirmado de COVID-19, foi colocado em uma terceira área (-Fig. 1). Todos os pacientes sintomáticos ou com histórico de contágio foram submetidos à triagem para COVID-19 por PCR. O resultado do exame foi disponibilizado em 72 horas

Além das três coortes diferentes na área de internação, na unidade de terapia intensiva (UTI), 10 leitos de isolamento foram reservados exclusivamente para pacientes com diagnóstico confirmado de COVID-19. Barreiras de segurança, com limites de transição, foram instaladas entre essas áreas. $\mathrm{O}$ acesso ao andar, onde ficava a área isolada para pacientes com COVID-19, foi bloqueado para funcionários que não fizessem parte da equipe escolhida para trabalhar no espaço.

\section{Reorganização dos Procedimentos Cirúrgicos}

Os procedimentos cirúrgicos foram realizados em dois centros cirúrgicos diferentes. Os pacientes assintomáticos foram encaminhados ao centro cirúrgico principal, com 18 quartos. Os pacientes com suspeita ou diagnóstico confirmado de COVID-19 foram encaminhados para outro centro cirúrgico, com três salas cirúrgicas em outro andar, totalmente independente do primeiro.

Tabela 1 Reorganização das atividades institucionais

\begin{tabular}{|c|c|}
\hline Atividades assistenciais & Atividades acadêmicas \\
\hline $\begin{array}{l}\text { - Liberação de profissionais com alto risco COVID-19 para } \\
\text { trabalho remoto. } \\
\text { - Redução do atendimento ambulatorial, com suspensão do } \\
\text { atendimento de novos pacientes. } \\
\text { - Separação das áreas de espera nas unidades ambulatoriais e } \\
\text { criação de um sistema escalonado de atendimento. } \\
\text { - Definição de área exclusiva para atendimento de pacientes e } \\
\text { colaboradores sintomáticos. } \\
\text { - Realocação e treinamento de profissionais para atuação nas } \\
\text { áreas mais críticas - Unidade de Terapia Intensiva (UTI), } \\
\text { Unidade Referenciada de Trauma e COVID-19 nas enfer- } \\
\text { marias. } \\
\text { - Disponibilização de iPads na UTI e enfermarias para que os } \\
\text { pacientes façam reuniões virtuais com seus familiares. } \\
\text { - Elaboração de manual sobre uso eficiente de equipamentos } \\
\text { de proteção individual (EPIs) e sua colocação na Área de } \\
\text { Infecção Hospitalar }\end{array}$ & $\begin{array}{l}\text { - Suspensão das atividades presenciais e adoção do formato } \\
\text { remoto para atividades em diversas áreas de especializa- } \\
\text { ção, como mestrado e reuniões clínicas. } \\
\text { - Utilização de manequins e simulação realista para treina- } \\
\text { mento de profissionais, inclusive de outras unidades de } \\
\text { saúde, sobre intubação, manejo ventilatório e fisioterapia } \\
\text { respiratória para COVID-19. } \\
\text { - Organização de escalas para manutenção das atividades em } \\
\text { laboratórios de pesquisa e biotério. }\end{array}$ \\
\hline
\end{tabular}




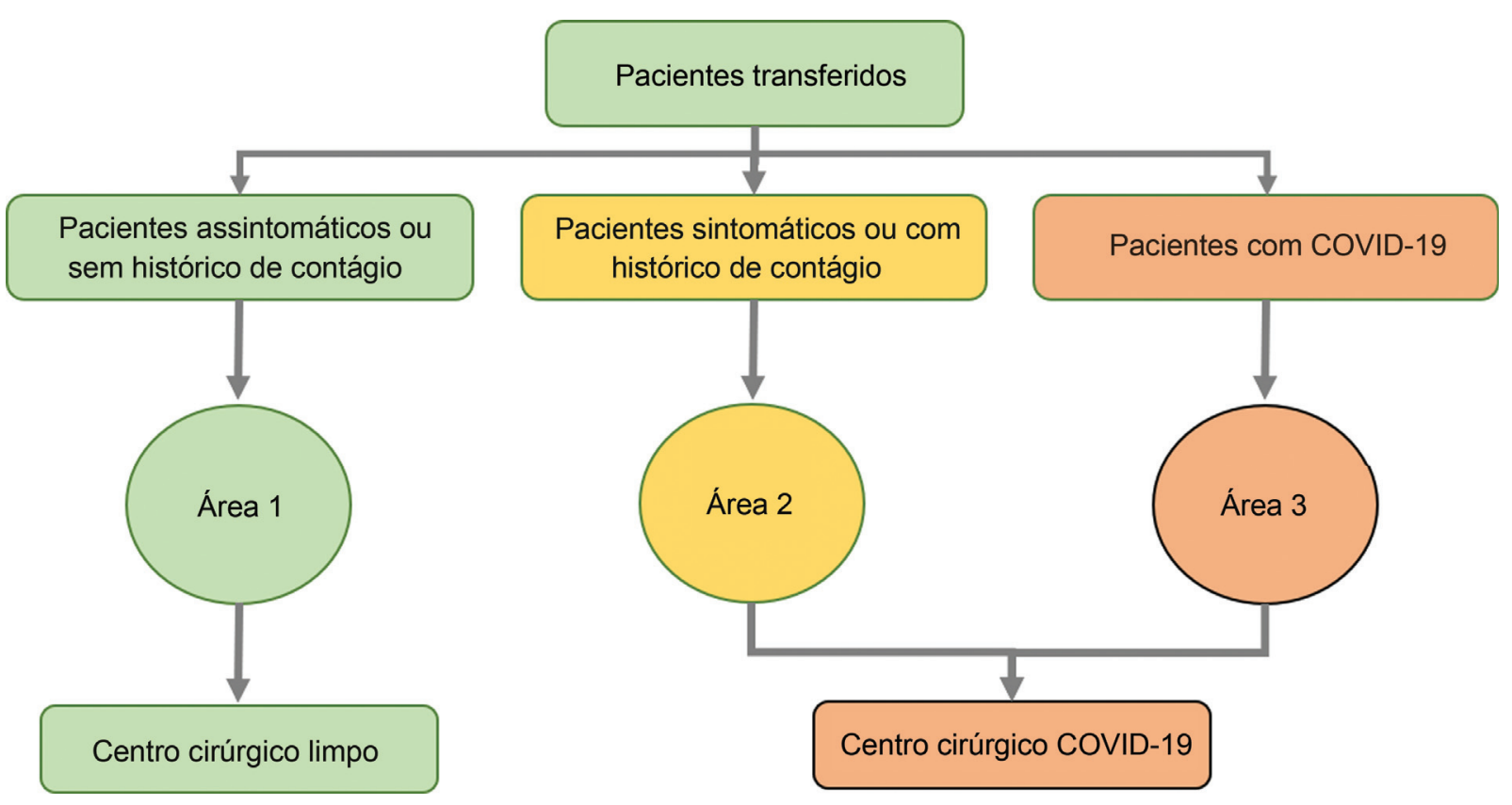

Fig. 1 Algoritmo para recepção e encaminhamento de pacientes transferidos.

O fluxo de atendimento nessas unidades foi alterado, com restrições ao número de profissionais permitidos durante a cirurgia. A equipe cirúrgica envolvida na realização do procedimento só teve acesso à sala de cirurgia após o término da anestesia.

\section{Análise de Dados}

A avaliação do efeito da implantação dos protocolos descritos foi feita por meio de comparação dos dados hospitalares de 16 de março de 2020 a 30 de junho de 2020 com informações do mesmo período de 2019. As variáveis analisadas foram: (i) o número de profissionais em licença, (ii) o número de cirurgias realizadas, (iii) atendimento ambulatorial, (iv) o número de transferências, (v) o tempo de internação hospitalar, (vi) o número de pacientes com diagnóstico de COVID-19 e a origem da infecção e (vii) o número de óbitos. O critério utilizado para caracterizar a infecção como comunitária ou proveniente de outra unidade de saúde foi o aparecimento de sintomas até o sétimo dia de internação. Após esse período, a infecção foi classificada como adquirida no instituto.

\section{Resultados}

No período estudado, 231 colaboradores, inclusive 160 profissionais de saúde, foram afastados de acordo com a Portaria $n^{\circ} 428$ do Ministério da Saúde que permitia o trabalho à distância para profissionais com mais de 60 anos, portadores de doenças crônicas e gestantes de alto risco.

As atividades acadêmicas dos cursos de pós-graduação stricto e lato sensu foram mantidas, com realização de aulas, encontros científicos e defesas de dissertações por meio de videoconferências.

À comparação de todos os dados anuais, a restrição à realização de procedimentos eletivos, tanto cirúrgicos quanto ambulatoriais, levou a uma redução de $48,5 \%$ na

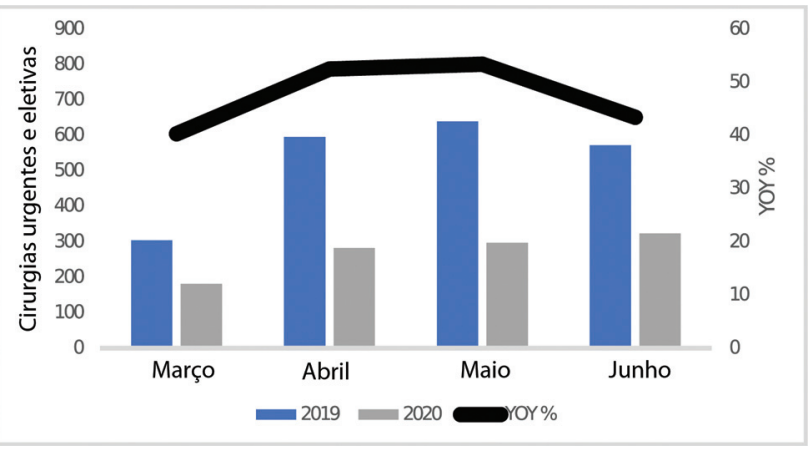

Fig. 2 Número de cirurgias de urgência e eletivas realizadas nos dois períodos avaliados. Azul: 2019. Cinza: 2020. Linha preta: variação ano a ano (em porcentagem).

produtividade cirúrgica (-Fig. 2) e a uma redução de $72,4 \%$ no atendimento ambulatorial em relação ao mesmo período de 2019 (-Fig. 3).

O novo papel assumido pelo instituto, $o$ atendimento de todos os pacientes com traumatismos ortopédicos de seu estado, levou a um aumento de $124,5 \%$ no número de transferências (-Fig. 4). 0 aumento das transferências foi acompanhado por um aumento de $63 \%$ no tempo de internação em abril e um aumento de mais de $100 \%$ em maio e junho de 2020 em comparação a 2019 (-Tabela 2).

O aumento do número de transferências também elevou o número de pacientes internados com diagnóstico de COVID-19 e dos casos de contaminação intra-hospitalar ( - Fig. 5). Além disso, devido à transmissão do vírus entre os profissionais do hospital, houve aumento do número de profissionais afastados por doença (-Fig. 5), principalmente enfermeiras e auxiliares de enfermagem no mês de abril ( - Tabela $\mathbf{3}$ ).

No período estudado, 631 pacientes de outras unidades foram transferidos para o instituto e 1.087 procedimentos cirúrgicos foram realizados. Entre os pacientes transferidos, 


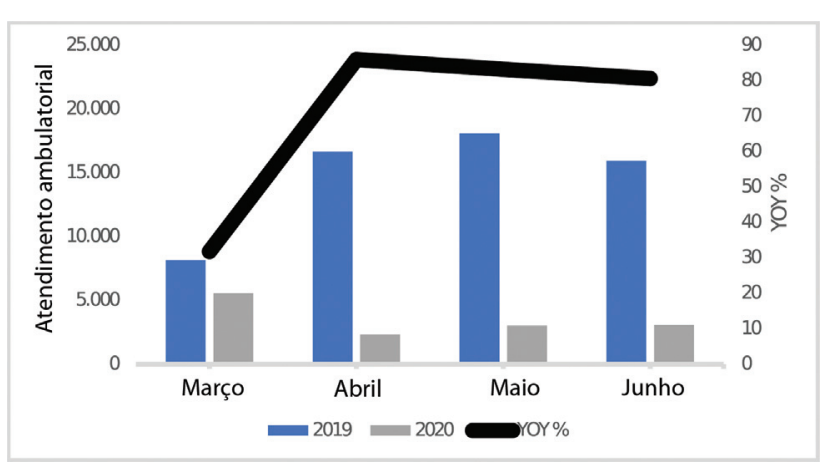

Fig. 3 Número de atendimentos ambulatoriais realizados nos dois períodos avaliados. Azul: 2019. Cinza: 2020. Linha preta: variação ano a ano (em porcentagem).

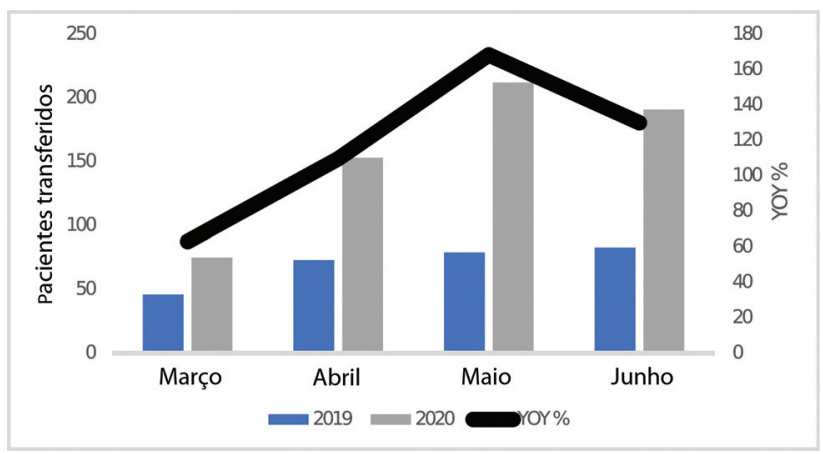

Fig. 4 Número de pacientes transferidos nos dois períodos avaliados. Azul: 2019. Cinza: 2020. Linha preta: variação ano a ano (em porcentagem).

Tabela 2 Duração da internação

\begin{tabular}{|l|l|l|l|}
\hline & $\begin{array}{l}\text { Número } \\
\text { médio de } \\
\text { dias em } \\
2019\end{array}$ & $\begin{array}{l}\text { Número } \\
\text { médio de } \\
\text { dias em } \\
2020\end{array}$ & $\begin{array}{l}\text { Variação ano } \\
\text { a ano (\%) } \\
\text { Aumento / } \\
\text { (Redução) }\end{array}$ \\
\hline $\begin{array}{l}\text { Março } \\
(16 \text { a } 31 \text { de } \\
\text { março) }\end{array}$ & 8,2 & 8,5 & $3,6 \%$ \\
\hline Abril & 9,1 & 14,9 & $63,7 \%$ \\
\hline Maio & 7,8 & 16,3 & $108,9 \%$ \\
\hline Junho & 7,7 & 16,6 & $114,1 \%$ \\
\hline
\end{tabular}

o diagnóstico de COVID-19 foi confirmado em 94 indivíduos, dos quais 46 pacientes tiveram o diagnóstico confirmado antes da transferência e 28 adquiriram COVID-19 no instituto. Nos 20 pacientes restantes, não foi possível rastrear a origem da infecção. Em outros 77 pacientes transferidos, apesar da suspeita, o diagnóstico de COVID-19 não foi confirmado pela PCR.

Durante o estudo, foram registrados 38 óbitos (em comparação a 11 no mesmo período de 2019), representando um aumento de $245 \%$. Entre os pacientes que faleceram, 31 (ou 81,5\%) tiveram diagnóstico confirmado de COVID-19.

\section{Discussão}

A disseminação do vírus SARS-CoV-2 foi rápida e extensa no Brasil, especialmente em estados altamente populosos, como

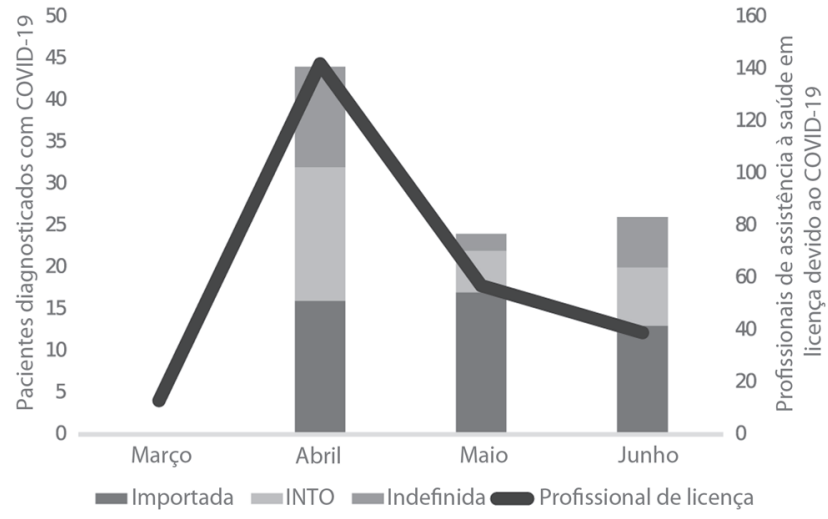

Fig. 5 Número de pacientes e profissionais com diagnóstico de COVID-19 e número de profissionais de saúde afastados. As barras representam o número de pacientes com COVID-19 classificados de acordo com a origem da infecção. A linha representa a quantidade de profissionais afastados devido à doença.

Tabela 3 Número de profissionais de saúde diagnosticados com COVID-19

\begin{tabular}{|l|l|l|l|l|}
\hline & Médicos & Enfermeiros & $\begin{array}{l}\text { Assistentes } \\
\text { de } \\
\text { enfermagem }\end{array}$ & Outros \\
\hline $\begin{array}{l}\text { Março } \\
(16 \text { a } 31 \\
\text { de março) }\end{array}$ & 10 & 0 & 3 & 0 \\
\hline Abril & 30 & 46 & 59 & 7 \\
\hline Maio & 11 & 14 & 19 & 13 \\
\hline Junho & 4 & 13 & 18 & 4 \\
\hline Total & 55 & 73 & 99 & 24 \\
\hline
\end{tabular}

Rio de Janeiro e São Paulo. Isso levou à necessidade de reorganização dos sistemas de saúde para redirecionar recursos e liberar leitos hospitalares para pacientes com COVID-19. Com esta nova diretriz, novas medidas e estratégias foram implementadas para as rotinas de recepção de pacientes, identificação de portadores de COVID-19, disponibilização de EPIs, controle da movimentação de profissionais de saúde e pacientes e alocação de salas cirúrgicas separadas para pacientes com ou sem COVID-19. ${ }^{4-6}$ Além dessas estratégias, foi preciso adotar medidas que permitissem a continuidade das atividades de ensino e pesquisa da forma menos danosa possivel. ${ }^{7-9}$

No período do estudo, 502 profissionais foram afastados de suas atividades laborais diárias por pertencerem a um grupo de alto risco ou serem diagnosticados com COVID-19. Isso causou uma redução de $21,6 \%$ no quadro de funcionários. Essa redução levou a um déficit representativo de recursos humanos em áreas críticas, principalmente aquelas que atendem à COVID-19. Assim, houve uma diminuição temporária na disponibilidade global de leitos devido à escassez desses profissionais. Cerca de $11 \%$ dos profissionais de saúde entraram em licença devido à infecção por COVID-19. Este número é inferior em comparação ao relatado em hospitais de Hong Kong e Itália, com $22 \%$ e $20 \%$, respectivamente. ${ }^{10}$ No entanto, o número de profissionais de saúde que foram 
infectados com COVID-19 pode ter sido subestimado, pois muitos casos que não puderam ser confirmados devido às limitações dos exames de COVID-19, em especial no início da pandemia.

A redução do número de consultas ambulatoriais e cirurgias foi um reflexo direto das medidas e protocolos implementados pelo Gabinete de Crise. A redução dessas atividades também tem sido adotada em outros serviços brasileiros e internacionais, destacando-se seu papel no auxílio à diminuição da disseminação do vírus. ${ }^{6,11}$

O papel central do Instituto em assumir o atendimento de todos os casos de traumatismo ortopédico do estado causou um aumento substancial no número de transferências e, consequentemente, na admissão de pacientes já contaminados pelo vírus em seu serviço. Em abril de 2020, 16 pacientes adquiriram COVID-19 durante o período de internação. Nos meses seguintes, com o reforço das medidas de segurança e higiene, embora o número de pacientes infectados transferidos fosse constante, houve uma queda drástica no número de pacientes que contraíram a doença no instituto, demonstrando a eficácia dos protocolos adotados.

O período de pico da primeira onda de COVID-19 no estado coincidiu com o maior número de pacientes infectados internados e com o menor número de profissionais de saúde devido ao vírus. Essa observação sugere que a disseminação da doença ocorreu não apenas entre os pacientes, mas também entre os profissionais de saúde. Vários fatores podem ter contribuído para a rápida disseminação do vírus entre os profissionais de saúde. Nguyen et al. ${ }^{12}$ mostraram que, apesar do uso de EPIs, o risco de contaminação dos profissionais de saúde pelo vírus SARS-CoV-2 foi três vezes maior em comparação à população em geral. Além disso, um estudo chinês de Guo et al. ${ }^{13}$ sobre cirurgiões ortopédicos relatou cansaço físico como outro fator de risco para contrair COVID-19. Esse mesmo estudo demonstrou que os locais mais prováveis de exposição ao vírus foram as enfermarias, os espaços públicos e os centros cirúrgicos. Tais estudos enfatizam a necessidade de adoção de outras medidas para o controle eficaz de infecções e reforçam a importância da gestão adequada de escalas e turnos para manter o equilíbrio mental dos profissionais de saúde.

Durante o estudo, 94 pacientes transferidos foram diagnosticados com COVID-19. É importante notar que, nos primeiros meses da pandemia, não havia testes diagnósticos suficientes à disposição e alguns pacientes suspeitos tiveram alta ou morreram sem confirmação diagnóstica. Isso sugere a subnotificação do número de casos de COVID-19 na unidade. A incidência de COVID-19 em pacientes atendidos no instituto foi maior em abril, com redução de $50 \%$ nos meses seguintes, principalmente devido ao controle efetivo da disseminação da doença.

No mesmo período, 631 transferências e 1.087 cirurgias foram realizadas, com média de internação de 16 dias ante oito dias no mesmo período de 2019. No instituto, as cirurgias eletivas são precedidas por rigoroso preparo préoperatório. Durante a pandemia, notou-se uma mudança no perfil dos pacientes atendidos, com aumento da gravidade dos quadros clínicos. $\mathrm{O}$ atendimento a esses pacientes, que muitas vezes necessitavam de intervenção médica urgente, levou à realização de procedimentos cirúrgicos sem o devido controle das comorbidades e descompensações clínicas do indivíduo. Por outro lado, se esses pacientes fossem diagnosticados com COVID-19, é provável que a cirurgia fosse adiada, levando à realização do procedimento após uma espera mais longa do que a atualmente recomendada na literatura. Estes dois fatores influenciam a recuperação pósoperatória dos pacientes e podem ter contribuído para o aumento dos tempos de internação, como já demonstrado. ${ }^{14-16}$ O diagnóstico de COVID-19 também pode ter contribuído para o aumento do tempo de internação, seja por agravar o quadro clínico do paciente e prolongar sua recuperação ou pela necessidade de cumprir os requisitos de quarentena durante o período de hospitalização.

A COVID-19 parece ser o principal fator que contribuiu para o aumento significativo do número de óbitos (245\%), uma vez que 31 dos 36 pacientes que faleceram eram casos confirmados da doença. Em nossos dados, a taxa de mortalidade de pacientes com COVID-19 foi de 32,9\%. Na literatura, a taxa de mortalidade de pacientes submetidos a procedimentos ortopédicos com diagnóstico perioperatório de COVID-19 é bastante variável, de 7,6\% a 43,7\%. ${ }^{17}$ Assim, ainda não há consenso quanto ao efeito da COVID-19 na mortalidade de pacientes submetidos a procedimentos ortopédicos de urgência. Embora alguns estudos afirmem que a taxa de mortalidade e o número de complicações são maiores em pacientes com COVID-19 e, portanto, que os procedimentos cirúrgicos deveriam ser adiados sempre que possível, ${ }^{18}$ outro conjunto de evidências sugere que, em pacientes idosos com fraturas de fêmur e diagnóstico de COVID-19, a cirurgia precoce teria melhores resultados, apesar da alta taxa de mortalidade. ${ }^{16-19}$ O conhecimento do efeito dos resultados cirúrgicos em pacientes com COVID-19 é de fundamental importância para médicos e gestores, permitindo o manejo adequado dos indivíduos que necessitam de procedimentos urgentes e orientando o desenvolvimento de protocolos para reintrodução de procedimentos eletivos com segurança.

\section{Conclusões}

A gestão de uma crise de saúde sem precedentes é um grande desafio para todos os provedores de serviços de saúde, mas também é uma oportunidade inestimável de aprendizado. A estratégia definida pelo instituto, com o apoio dos órgãos de controle, de transformar um centro de cirurgias eletivas de alta complexidade em um hospital de trauma durante a pandemia de COVID-19, permitiu a manutenção de sistemas operacionais e resilientes, assegurando o atendimento seguro e de qualidade de pacientes com traumatismo ortopédico de todo o estado.

Embora este estudo seja limitado por seu desenho retrospectivo e as ações e protocolos descritos façam parte de um conjunto amplo de medidas que visam à reorganização do sistema de saúde de uma única unidade federativa durante a pandemia de COVID-19, os resultados apresentados representam a realidade de apenas uma instituição. No entanto, novos estudos devem ser realizados para avaliar o efeito das estratégias e protocolos implementados pelo Gabinete de Crise nas atividades de outros hospitais da região. 
Conflitos de Interesse

Os autores não têm conflitos de interesse a declarar.

\section{Referências}

1 World Health Organization. WHO announces COVID-19 outbreak a pandemic? 2020. [Accessed 22 June 2020] from: http://www. euro.who.int/en/health-topics/healthemergencies/coronaviruscovid-19/news/news/2020/3/whoannounces-covid-19-outbreak -a-pandemic

2 Brazil. Ministry of Health Brazil confirms first case of the disease. 2020. [Accessed 2 August 2020] from: https://www.saude.gov.br/ noticias/agencia-saude/46435-brasil-confirma-first-case-of-newcoronavirus

3 Iacobucci G. Covid-19: all non-urgent elective surgery is suspended for at least three months in England. BMJ 2020;368:m1106

4 Ruggieri P, Trovarelli G, Angelini A, Pala E, Berizzi A, Donato D. COVID-19 strategy in organizing and planning orthopedic surgery in a major orthopedic referral center in an area of Italy severely affected by the pandemic: experience of the Department of Orthopedics, University of Padova. J Orthop Surg Res 2020;15 (01):279

5 Casiraghi A, Domenicucci M, Cattaneo S, et al. Operational strategies of a trauma hub in early coronavirus disease 2019 pandemic. Int Orthop 2020;44(08):1511-1518

6 Lima EBS, Belangero PS, Falótico GG, Mansur NSB, Luzo MVM, Reis FBD. Intervention Protocol of the Orthopedics and Traumatology Department of a High-Complexity University Hospital to Cope with the COVID-19 Pandemic. Rev Bras Ortop (Sao Paulo) 2020;55 (03):269-277

7 Awad ME, Rumley JCL, Vazquez JA, Devine JG. Perioperative Considerations in Urgent Surgical Care of Suspected and Confirmed COVID-19 Orthopaedic Patients: Operating Room Protocols and Recommendations in the Current COVID-19 Pandemic. J Am Acad Orthop Surg 2020;28(11):451-463

8 Mavrogenis AF, Quaile A, Scarlat MM. The virus crisis affects Orthopaedic surgery and scientific activities worldwide. Int Orthop 2020;44(05):813-817
9 Kogan M, Klein SE, Hannon CP, Nolte MT. Orthopaedic Education During the COVID-19 Pandemic. J Am Acad Orthop Surg 2020;28 (11):e456-e464

10 Nagesh S, Chakraborty S. Saving the frontline health workforce amidst the COVID-19 crisis: Challenges and recommendations. J Glob Health 2020;10(01):010345

11 Nuñez JH, Sallent A, Lakhani K, et al. Impact of the COVID-19 Pandemic on an Emergency Traumatology Service: Experience at a Tertiary Trauma Centre in Spain. Injury 2020;51(07):1414-1418

12 Nguyen LH, Drew DA, Joshi AD, et al. Risk of COVID-19 among frontline healthcare workers and the general community: a prospective cohort study. medRxiv 2020;2020:x

13 Guo X, Wang J, Hu D, et al. Survey of COVID-19 Disease Among Orthopaedic Surgeons in Wuhan, People's Republic of China. J Bone Joint Surg Am 2020;102(10):847-854

14 Gholson JJ, Noiseux NO, Otero JE, Gao Y, Shah AS. Patient Factors Systematically Influence Hospital Length of Stay in Common Orthopaedic Procedures. Iowa Orthop J 2017;37:233-237

15 Egol KA, Konda SR, Bird ML, et al; NYU COVID Hip Fracture Research Group. Increased Mortality and Major Complications in Hip Fracture Care During the COVID-19 Pandemic: A New York City Perspective. J Orthop Trauma 2020;34(08):395-402

16 Muñoz Vives JM, Jornet-Gibert M, Cámara-Cabrera J, et al; Spanish HIP-COVID Investigation Group. Mortality Rates of Patients with Proximal Femoral Fracture in a Worldwide Pandemic: Preliminary Results of the Spanish HIP-COVID Observational Study. J Bone Joint Surg Am 2020;102(13):e69

17 Kumar Jain V, Lal H, Kumar Patralekh M, Vaishya R. Fracture management during COVID-19 pandemic: A systematic review. J Clin Orthop Trauma 2020;11(Suppl 4):S431-S441

18 Doglietto F, Vezzoli M, Gheza F, et al. Factors Associated With Surgical Mortality and Complications Among Patients With and Without Coronavirus Disease 2019 (COVID-19) in Italy. [published online ahead of print, 2020 Jun 12]JAMA Surg 2020;155(08):1-14

19 Sobti A, Memon K, Bhaskar RRP, Unnithan A, Khaleel A. Outcome of trauma and orthopaedic surgery at a UK District General Hospital during the Covid-19 pandemic. J Clin Orthop Trauma 2020;11(Suppl 4):S442-S445 